\title{
MAC Protocol Switching: A Novel Tool for \\ Performance Adaptation in Wireless Sensor Networks
}

\author{
Fan Yu and Subir Biswas \\ Electrical and Computer Engineering \\ Michigan State University, USA \\ \{yufan,sbiswas\}@egr.msu.edu
}

Received: February 1, 2012

Accepted: May 16, 2010

Published: May 30, 2010

DOI: $10.5296 /$ npa.v4i1.1347

URL: http://dx.doi.org/10.5296/npa.v4i1.1347

\begin{abstract}
This paper presents a distributed MAC protocol switching mechanism for maximizing network throughput in the presence of traffic and topology heterogeneity. The key idea behind dynamic MAC switching is for each node to use its local topology and traffic density information to decide the most suitable MAC protocol that can maximize the MAC layer throughout in the neighborhood. A formal MAC switching rule is developed using analytical formulation of the MAC throughput available in the literature. NS2 based simulation experiments demonstrate that with the proposed MAC switching strategy, nodes in a wireless sensor network are able to achieve maximum MAC throughput by adaptively choosing the appropriate MAC protocol in the presence of heterogeneity in terms of data rate and node population.
\end{abstract}

Keywords: Medium Access Control, Self-Organization, Traffic Heterogeneity, Protocol Switching 


\section{Introduction}

\subsection{Background}

Wireless sensor networks are motivated by applications such as intrusion detection, battle-field surveillance, and environment and health monitoring. Although there have been significant recent innovations at different protocol layers, maximizing network throughput at the MAC layer in a multi-mission network is still a key design challenge. The traffic heterogeneity in a multi-mission network can manifest through: a) spatial and temporal variance in data rates [1] across different parts of a network supporting different applications, and b) temporal variance in number of active transmitters at a given time and at a given part of the network. The problem we address in this paper is how to develop a self-organizing MAC layer that can maximize network throughput in the presence of the above traffic and network heterogeneity.

\subsection{Related work}

The existing MAC layer self-organization can be divided into two broad categories, namely, intra-MAC and inter-MAC approaches. The intra-MAC approaches include contention-based [2-4] and schedule-based [5-7] protocols. The intra-MAC self-organization is achieved by dynamically adjusting the state machine and parameters of a specific MAC protocol. CSMA/CA [2] and TDMA [5-7] are two examples of such intra-MAC approaches. CSMA/CA has the advantages of simplicity, flexibility and robustness, and it does not need any infrastructure support or clock synchronization. The most notable advantage of CSMA/CA is that a node can access all available wireless bandwidth in its neighborhood in an as-needed manner. However, the medium access collisions and the corresponding unbounded access delay are a concern for CSMA/CA and all other protocols in that category, mainly because of their underlying random access. TDMA protocols, on the other hand, allocate fixed, collision-free, and guaranteed bandwidth for all nodes in the network. While guaranteeing maximum delay bounds, the primary disadvantage of TDMA is that it does not perform well under dynamic bandwidth requirements. In other words, TDMA performs well under highly symmetrical load, but performs poorly under asymmetric load. Under the latter situation, CSMA based protocols outperform TDMA.

The authors in [8] propose an inter-MAC self-organization called Funneling-MAC. This is designed to address the traffic funneling effect near sink nodes caused by gradual aggregation of multipoint-to-point traffic. With Funneling-MAC, nodes near a sink (i.e. those with heavy traffic) are said to belong within an intensity area and they run a TDMA protocol which is managed by the sink node. Nodes outside the intensity area (i.e. those with relatively lighter traffic) run CSMA without any coordination from the sink. The Funneling-MAC [8] provides beacon based protocol syntaxes that the sink uses for dynamically deciding the boundary of the intensity area. Through a dynamic depth-tuning algorithm, the network throughput is maximized and the packet loss rate is minimized at the sink point. Although it provides a novel way of accomplishing inter-MAC (i.e. between CSMA and TDMA) self-organization, one notable limitation of Funneling-MAC is that it is suitable only for multipoint-to-point applications and not for peer-to-peer traffic, which is often required by sensor applications with distributed data fusion requirements. 


\subsection{Proposed Dynamic MAC Protocol Switching}

In the proposed approach in this paper the operating MAC protocol within a node is dynamically switched between CSMA and TDMA based on the instantaneous traffic and topological property of the neighborhood of the node. The mechanism is designed to be general so that unlike Funneling-MAC [8], it can be applied for handling both multipoint-to-point and peer-to-peer data traffic. Each node monitors its neighborhood traffic and topology conditions, determines the appropriate individual MAC protocol to run, and switches its protocol as needed.

The contributions of the paper are as follows. First, it proposes a self-organizing MAC protocol switching paradigm to address the problem of how to achieve the maximum throughput in a network with traffic heterogeneity. Second, it introduces syntax extensions to the protocols CSMA/CA and TDMA so that they can co-exist in immediately neighboring nodes. Third, it develops dynamic protocol switching rules based on an analytical model. Finally, a detailed simulation model is developed for experimentally validating the concept of dynamic MAC protocol switching in wireless networks.

\section{Protocol Adaptations}

The key idea behind dynamic protocol switching is that depending on the specific traffic pattern in its neighborhood, a node can choose to run one of multiple MAC protocols. This capability in a network can lead to situations in which a node can have multiple neighbors that are running different protocols. This will require the node in question to be able to communicate using multiple different MAC protocols simultaneously in order to maintain end-to-end network connectivity. This MAC coexistence can be particularly challenging due to the fact that a sensor node needs to accomplish this over as single wireless interface. In this paper, coexistence of two MAC protocols, namely a random access CSMA/CA and a schedule based TDMA, are studied. CSMA/CA and TDMA are chosen because they serve as good representations of contention based and schedule based protocols respectively.

\subsection{Adaptations of CSMA/CA for TDMA Coexistence}

The objective here is to adapt the CSMA/CA protocol syntaxes such that a node running CSMA/CA can also communicate with a node running TDMA. The basic RTS-CTS-DATA cycle in CSMA/CA transmissions is maintained. The Acknowledgement (ACK) is not utilized, (meaning there is no link layer), and the regular exponential back-off are still applied. Adaptations of the CSMA/CA syntaxes are made such that no access collisions are imposed on the TDMA nodes in a CSMA/CA node's neighborhood.

Adapted Send Logic: A CSMA/CA sender is able to estimate the duration of a successful transmission in terms of RTS, CTS, the data message, and the various inter frame spacing. If it knows the TDMA slots of all its neighbors, then the sender can determine if the following data transmission will overlap with the neighbors' TDMA slots. If there is at least one anticipated overlapping, the sender simply defers its transmission till the end of all its neighbors' TDMA slots. Otherwise, the data transmission could carry on as usual. 


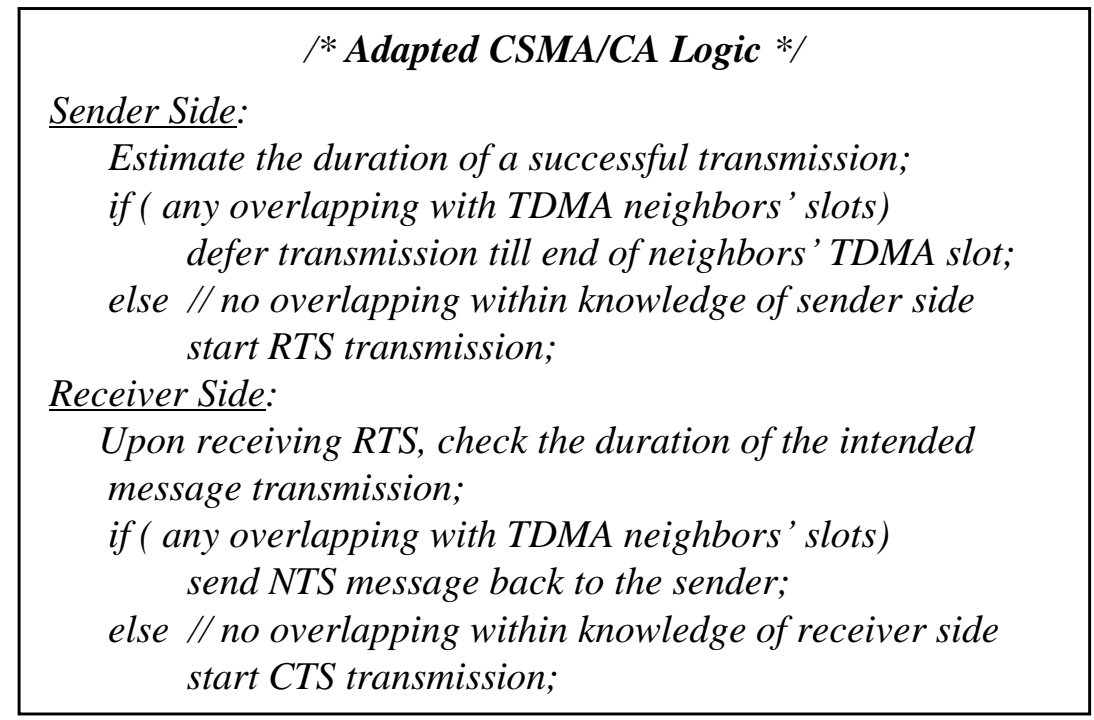

Fig. 1: Pseudo code for adapted CSMA/CA logic

Adapted Receive Logic: Upon receiving an RTS message, from its NAV field, the intended receiver is able to know the expected duration of the following data message. If that expected duration overlaps with the TDMA slots of the receiver's TDMA neighbors, the receiver will send a Not-to-Send (NTS) message, containing the duration of the overlapping period, back to the sender. Upon receiving the NTS message, the sender will then decide as to when to restart the current data transmission in order to avoid the slots of all TDMA neighbors of the sender as well as the receiver. The logic of this adapted CSMA/CA is presented in the pseudo code in Fig. 1.

\subsection{Adaptation of TDMA for CSMA/CA Coexistence}

The same slot and frame structure as in regular TDMA is maintained. Meaning, each TDMA node is allocated a slot in each frame, which is periodic. As an adaptation, an RTS-CTS syntax is added within the TDMA slots so that before sending a data message, a node needs to perform the RTS/CTS handshake with the receiver node. This is done irrespective of whether the receiver is currently using CSMA/CA or TDMA.

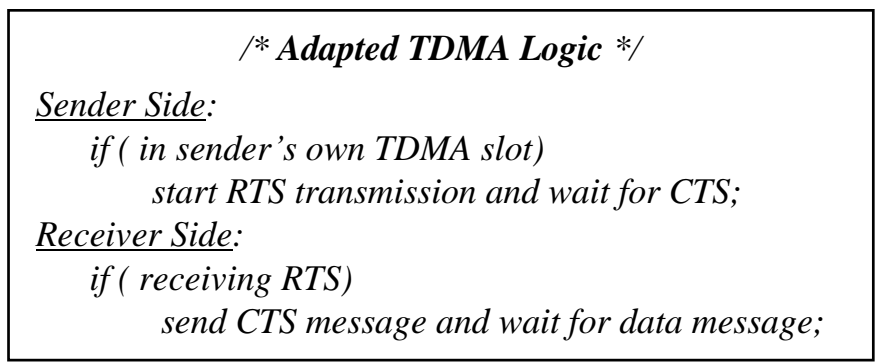

Fig. 2: Pseudo code for adapted TDMA logic 
The addition of this RTS-CTS within TDMA allows the node to send data to both CSMA/CA and TDMA nodes. Note that a TDMA sender node does not need to sense the channel before it sends the CTS since all nodes in its neighborhood (CSMA/CA or TDMA) respect the TDMA slot allocation and the channel is guaranteed to be free after the sender's slot has started. The RTS-CTS mechanism, in fact, is used to make sure that the receiver node is in a state to receive. The pseudo code for the adapted TDMA logic is shown in Fig. 2.

\subsection{Transmission Rules}

\subsubsection{Nodes with Same MAC Protocol}

This scenario refers to when nodes within two hop neighborhood run the same MAC logic. In an all-CSMA/CA neighborhood, nodes execute modified CSMA/CA logic with an effective behavior of regular CSMA/CA. In an all-TDMA neighborhood, the RTS/CTS extensions of the TDMA logic are used for incorporating future arrival of CSMA nodes.

\subsubsection{Transmissions From TDMA to CSMA/CA}

As shown in Fig. 3, when a TDMA node intends to transmit to a neighboring CSMA/CA node, an RTS message is first sent out in the beginning of the transmitter's allocated slot. Upon reception of the RTS, the CSMA/CA receiver sends a CTS message back in response. Once the TDMA node receives the CTS, data packet transmission follows in the remaining slot time. Note that the TDMA nodes attempt to send data only during their assigned TDMA slots. Although insignificant compared to the data packet length, the RTS/CTS exchange at the beginning of each slot of the TDMA nodes is regarded as an overhead.

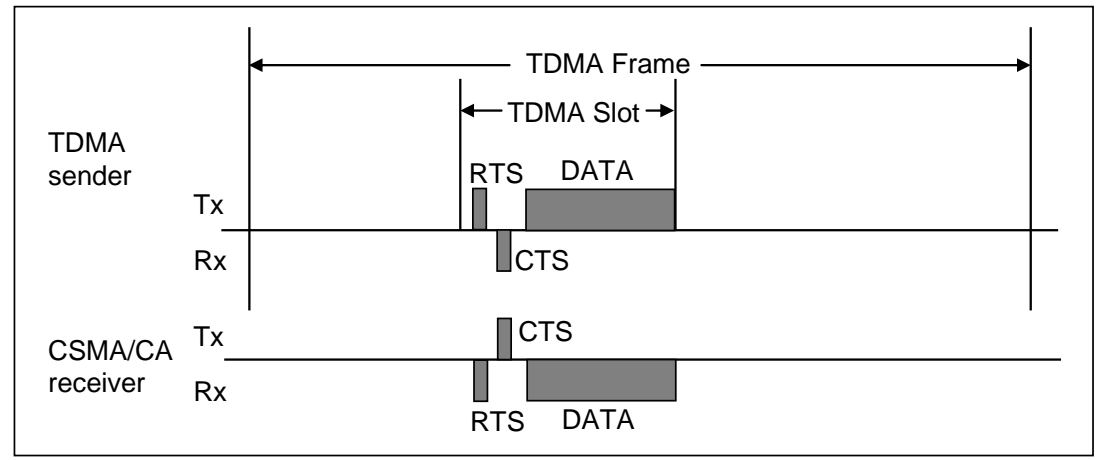

Fig. 3: Transmission from a TDMA node to a CSMA/CA node

\subsubsection{Transmissions From CSMA/CA to TDMA}

Transmission rules for CSMA/CA to TDMA data transmissions are relatively more complex. Ideally, assuming successful carrier sensing, a CSMA/CA node should be able to transmit asynchronously; that is when there is a message to be sent without the notion of any slot constraints. However, in this design, when a CSMA/CA node transmits to a TDMA neighbor, transmission needs to be partially synchronous. With the fully asynchronous approach, there is a chance that the transmission from a CSMA/CA node may fully or partially collide with the transmission of a neighboring TDMA node in its assigned slot.

Collisions at the CSMA/CA nodes can be handled via the usual back-off retransmissions. However, collisions at the TDMA nodes can be more of a problem, because after a TDMA 
node collides its earliest retransmission can be during the next frame, which is quite expensive in terms of delay. Considering this, higher transmission priority is given to the TDMA nodes when both TDMA and CSMA/CA nodes attempt to access the medium simultaneously. Such priority is achieved in the following two ways.

(1) A CSMA/CA node is never allowed to interfere with the potential transmissions of up to 2-hop TDMA neighbors. This requires such a transmission to end before the beginning of the next TDMA slot. The owner of the next slot can be a neighbor of either the CSMA/CA sender itself, or a neighbor of the receiver of the current transmission, which is a TDMA node in this case. In order to make sure that both cases are handled, the following measures are taken for three different scenarios.

Scenario-1: As shown in Fig. 4, at the CSMA/CA side, if a message transmission (including RTS/CTS) cannot end before the start of the immediately next TDMA neighbor's slot, then the CSMA/CA node should defer the transmission after the TDMA neighbor's slot.

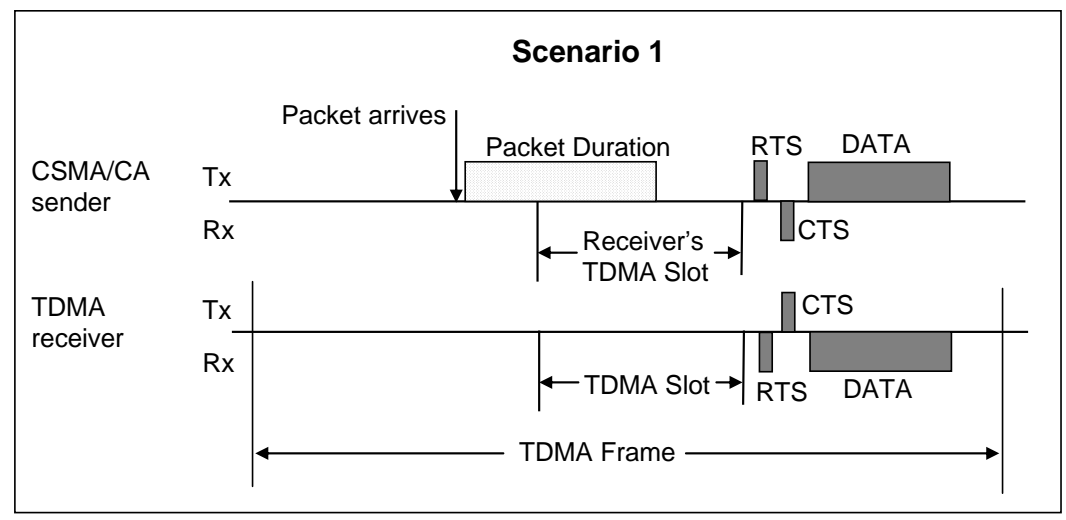

Fig. 4: Transmission from a CSMA/CA node to a TDMA node in Scenario-1

Scenario-2: As shown in Fig. 5, after an RTS reception, if a TDMA node finds out that the interval between the current time and the earliest next TDMA neighbor's slot is smaller than the CTS message duration, then the CTS will not be sent back to the CSMA/CA node. Instead, an NTS (Not-to-Send) message will be sent back to the CSMA/CA node.

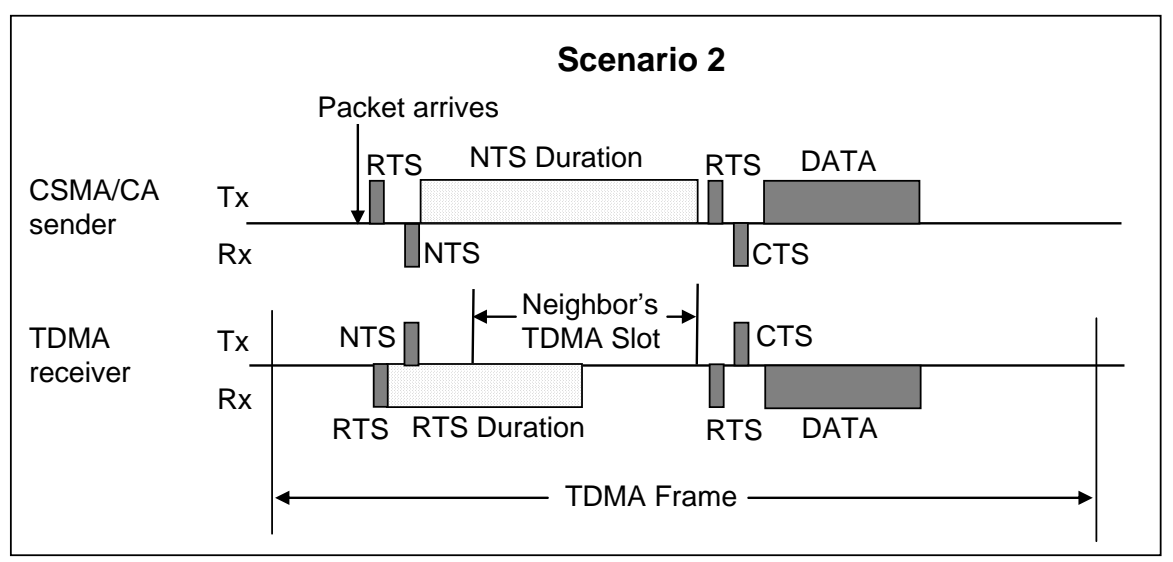

Fig. 5: Transmission from a CSMA/CA node to a TDMA node in Scenario-2 


\section{Macrothink}

Scenario-3: Upon reception of a CTS message, the corresponding data message could be transmitted if no violation of scenario-1 was found. In this case, at the sender, no NTS message is received for deferment of a transmission. A CSMA/CA node in this scenario still checks the validity of scenario-1 after receiving a CTS message. This redundancy is to ensure the priority of TDMA nodes over CSMA/CS nodes in the presence of network topology dynamics. Fig. 6 demonstrates the activities in Scenario-3.

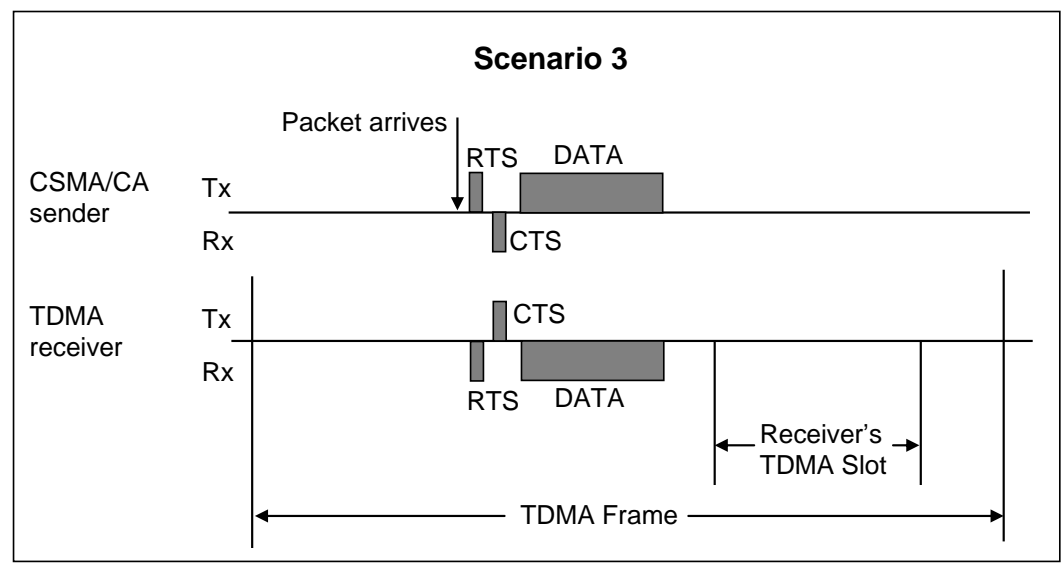

Fig. 6: Transmission from a CSMA/CA node to a TDMA node in Scenario 3

(2) Usage of a TDMA neighbor's slots by a CSMA/CA node is allowed only when the TDMA neighbors do not have anything to send. This is accomplished by having a randomly distributed RTS deferring time for the CSMA/CA nodes when current time is in their TDMA neighbors' slots.

\section{MAC Protocol Switching Logic}

\subsection{Influencing Switching Parameters}

Consider a network of $n$ nodes and each node's MAC layer is modeled as an M/M/1 queue. If $\lambda$ and $\mu$ represent the data rate and the MAC layer service rate at each node, the unsaturated network-wide throughput can be written as $n \cdot \lambda$, and the saturated network-wide throughput can be expressed as $n \cdot \mu$. The network-wide throughput of a fully connected $n$-node TDMA network can be written as [9]:

$$
S_{T D M \overline{\bar{A}}} f(\lambda, n, F),
$$

where $F$ is the TDMA frame duration which is fixed for a given network. Similar throughput for a CSMA/CA network can be written as [10-15]:

$$
S_{C S M A / C A}=g\left(\lambda, n, W_{\min }, m\right),
$$

$W_{\min }$ is the minimum contention window size for CSMA/CA, and $m$ is the maximum 
back-off stage.

Therefore, the throughput of a network with $n_{T D M A}$ and $n_{C S M A / C A}$ number of nodes running the protocols TDMA and CSMA/CA respectively is:

$$
S=S_{T D M A}+S_{C S M A / C A}=f\left(\lambda, n_{T D M A}, F\right)+g\left(\lambda, n_{C S M A / C A}, W_{\mathrm{min}} m\right)
$$

For a given network, the quantities $F, m$ and $W_{\min }$ are all constants. Thus, the throughput is decided based only on load $\lambda$ and the node counts in Eqn. 3.

Implications of different data rates $(\lambda)$ :The maximum MAC layer service rate of a node running TDMA is bounded by the TDMA frame duration, and can be reported as a fixed quantity $\mu_{M A X-T D M A}$. Meaning, as long as the data rate $\lambda$ at a node is less than or equal to $\mu_{\text {MAX-TDMA }}$, the MAC layer throughput is same as the load $\lambda$.

For a node running CSMA/CA, however, there is no such absolute bound for the achievable throughput. The per-node throughput here depends on the amount of MAC layer collisions contributed by other CSMA/CA nodes in the neighborhood. In other words, the MAC throughput of a CSMA/CA node is less than or equal to the MAC load $\lambda$; the exact value depends on the degree of collisions.

Implications of the number of active nodes $(n)$ : If $S_{\text {single-TDMA }}$ represents the MAC throughput of a single node running TDMA MAC, then the aggregated throughput of a network cluster of $n$ active nodes running TDMA can be expressed as $n \cdot S_{\text {sin } g l e-T D M A}$. For CSMA/CA, however, the aggregated throughput of the cluster is less than $n \cdot S_{\text {single-CSMA/CA }}$, since with $n$ nodes running CSMA/CA in a cluster reduces the effective MAC throughput (due to contentions and collisions) of each node to be less than $S_{\text {single-CSMA/CA }}$. Such throughout reduction does not apply for TDMA because of the lack of bandwidth contention and collisions.

The above analysis indicates that in order to maximize the aggregated MAC layer throughput of a network, the MAC protocols for each node should be individually chosen CSMA/CA or TDMA) based on its data load $\lambda$ and the number of active nodes $n$ in its immediate neighborhood.

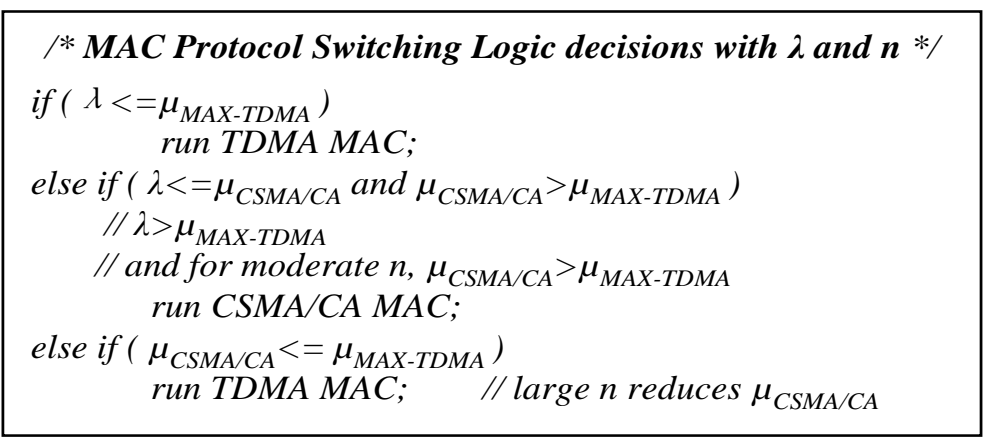

Fig. 7: Logic for protocol switching decision with $\lambda$ and $n$ 


\subsection{Switching Criteria}

According to the analysis presented in Section 3.1, when for a node the condition $\lambda \leq \mu_{M A X-T D M A}$ is true, the node should choose TDMA as the MAC protocol for the best throughput. That is irrespective of the number of active neighbors $n$. When $\lambda>\mu_{\text {MAX-TDMA }}$, the quantity $n$ needs to be considered. If the effective MAC service rate $\mu_{C S M A / C A}$ at the current $n$ for the node is smaller than the constant $\mu_{M A X-T D M A}$, then also TDMA should be chosen. Finally, if for the current $n$, the quantity $\mu_{C S M A / C A}$ is larger than $\mu_{M A X-T D M A}$ then CSMA/CA should be chosen for maximizing the MAC throughput. The protocol switching criteria is summarized in the pseudo code in Fig. 7.

In the above decision process, the rate $\lambda$ can be measured locally, and the quantity $\mu_{M A X-T D M A}$ is dimensioned based on the preset frame duration. The only unknown quantity is $\mu_{C S M A / C A}$ which needs to be computed as a function of all system parameters, especially considering the number of active neighbors $n$. It can be computed from the following analysis [10-15] which involves solving a set of equations as follows.

$$
\begin{gathered}
\frac{1}{b_{0,0}}=\left\{\begin{array}{c}
\frac{2 p W\left[1-(2 p)^{m}\right](1-p)+p(1-2 p)\left(1-p^{m}\right)}{2(1-2 p)(1-p)}+\frac{\left(2-p_{b}\right)(W+1)}{2}+\frac{1-p_{b}}{p_{a}}, m \leq m^{\prime} \\
\frac{2 p W\left[1-(2 p)^{m^{\prime}}\right](1-p)+p(1-2 p)\left(1-p^{m^{\prime}}\right)}{2(1-2 p)(1-p)}+\frac{\left(2-p_{b}\right)(W+1)}{2}+\frac{1-p_{b}}{p_{a}} \\
+\frac{p^{m^{\prime}+1}\left(W_{\max }+1\right)\left(1-p^{m-m^{\prime}}\right)}{2(1-p)}, m>m^{\prime}
\end{array}\right. \\
\tau=\sum_{i=0}^{m} b_{i, 0}=\frac{1-p^{m+1}}{1-p} b_{0,0} \\
p=1-(1-\tau)^{N-1} \\
1 / \mu=\frac{1-p-p(2 p)^{m}}{1-2 p} \frac{W}{2} \sigma+t_{x}+t_{c} \frac{p}{1-p}+\rho(N-1)\left(t_{x}+t_{c} \frac{p}{1-p}\right) \\
p_{a}=1-e^{-\lambda \sigma} \\
p_{b}=\rho=\lambda / \mu \\
\mathbf{t}_{\mathbf{x}}=\mathbf{R T S}+\text { SIFS }+ \text { CTS }+ \text { SIFS }+ \text { Header }+ \text { DATA + DIFS } \\
\mathrm{t}_{\mathrm{c}}=\mathrm{RTS}+\mathrm{DISF}
\end{gathered}
$$


where $m$ is the maximum number of retransmissions, $m$ ' is the maximum number of back-off stages, $\sigma$ is the slot duration of CSMA/CA, $W$ is the minimum congestion window size, $N$ is the number of contenders, $\lambda$ is the data rate and $\mu$ is the MAC service rate. The quantity $\mu_{C S M A / C A}$ can be obtained from Eqn. 9 after the above system of equation (Eqns. 4 through 11) is solved [10-15].

\section{Experimental Evaluation}

The proposed protocol switching logic has been implemented in a 100-node sensor network within the NS2 simulator [16]. A wireless data rate of $2 \mathrm{Mbps}$ has been chosen with fixed packet duration of $2 \mathrm{~ms}$, which is also the TDMA slot duration. Unless stated otherwise, we have fixed the TDMA frame size to 20 slots for all experiments. A local traffic model is used in which each node generates packets at a pre-specified rate ( $\lambda$ packets per second). No end-to-end routing is considered for this evaluation. The numbers of active nodes and data generation rate have been varied. Before running protocol switching logic, we run a distributed TDMA protocol ISOMAC [17] to assign TDMA slots to the network nodes. The other baseline parameters for the simulation are shown in Table 1.

\begin{tabular}{|l|l|}
\hline \multicolumn{2}{|c|}{ TDMA MAC Related } \\
\hline Frame Size (number of slots) & $\mathbf{2 0}$ \\
\hline TDMA Slot Duration & $\mathbf{2 m s}$ \\
\hline \multicolumn{2}{|c|}{ CSMA/CA MAC Related } \\
\hline Minimum Congestion Window Size & $\mathbf{3 2}$ \\
\hline Maximum Number of Back-off Stage & $\mathbf{5}$ \\
\hline CSMA/CA Slot size & $\mathbf{2 0 u s}$ \\
\hline Maximum Number of Retransmission & $\mathbf{7}$ \\
\hline SIFS Duration & $\mathbf{1 0}$ us \\
\hline DIFS Duration & $\mathbf{3 0}$ us \\
\hline \multicolumn{2}{|c|}{ Protocol Switching Related } \\
\hline Evaluation Time & $\mathbf{W = 3}$ \\
\hline Percentage of Decision Count & D=55\% \\
\hline
\end{tabular}

Table 1: Baseline experimental parameters in simulation

\subsection{Switching in Response to Changes in Data Rate}

As presented in Section 3.1, since the data rate $\lambda$ is a key criterion for protocol switching, its variation in a network neighborhood is expected to trigger such switching for maintaining high network throughput. In practice, the data rate variation in wireless sensor network can be caused by several factors including start or termination of applications and changes in application data rates. In the simulated 100-node sensor network, at any given time each node sends packets to one of its 1-hop neighbors at a given rate. Such rates are changed from 100 packets/second to 20 packets/second at time 21.0 second, and then changed back again at time 31.0 second. The corresponding network dynamics in terms of MAC protocol switching 
and its impacts on the network throughput are shown in Fig. 8.

In addition to the data rate graph (the solid line) for each node, Fig. 8 reports three other lines representing network-wide throughout for three distinct operating situations. The first one demonstrates the network throughput when all nodes run CSMA/CA. The second one shows what happens when all nodes run TDMA. Finally, the one marked switching, demonstrates the network throughput in the presence of adaptive MAC protocol switching following the logic as described in Section 3.

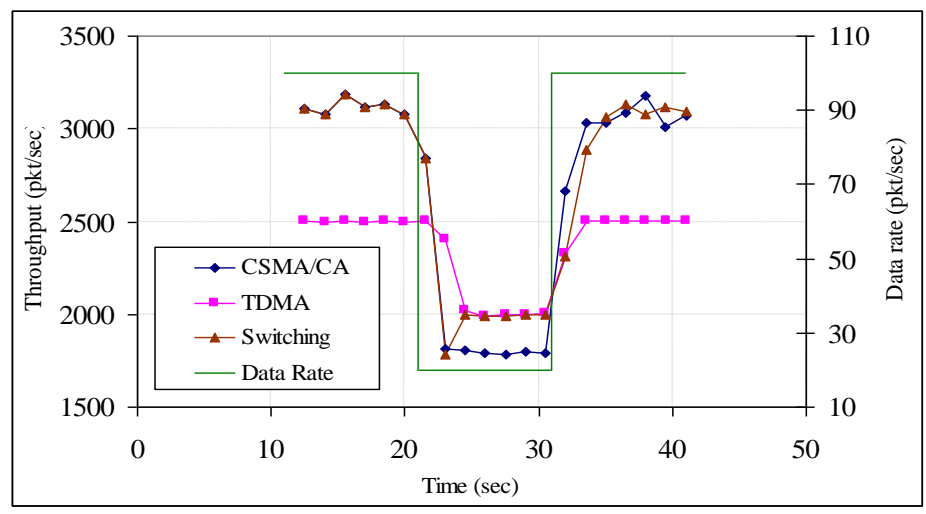

Fig. 8: Impacts of protocol switching with varying data rate

As can be seen in Fig. 8, initially when the data rate is high, the CSMA/CA mode of operation provides better effective throughput than the TDMA mode since the TDMA throughput is bounded by the frame size. Note, however, that the CSMA/CA mode throughput is lower than the maximum possible throughput, indicating certain amount of throughput loss due to the CSMA/CA contention and collisions. These contention and collisions for CSMA/CA persist even when the data rate is lowered (at time $20 \mathrm{sec}$.). At lower rates, however, TDMA can sustain the offered load since it is able to provide completely collision free MAC access to the channel. As a result, as evident from Fig. 8, the TDMA mode of operation provides better network-wide throughput at lower loading conditions.

The throughput line representing protocol switching demonstrates that via exercising such MAC layer switching, the network is able to track the best possible throughput (those of CSMA/CA at high load, and TDMA at low load) as a response to the change in loading conditions. This partially validates the switching logic presented in Section 3.

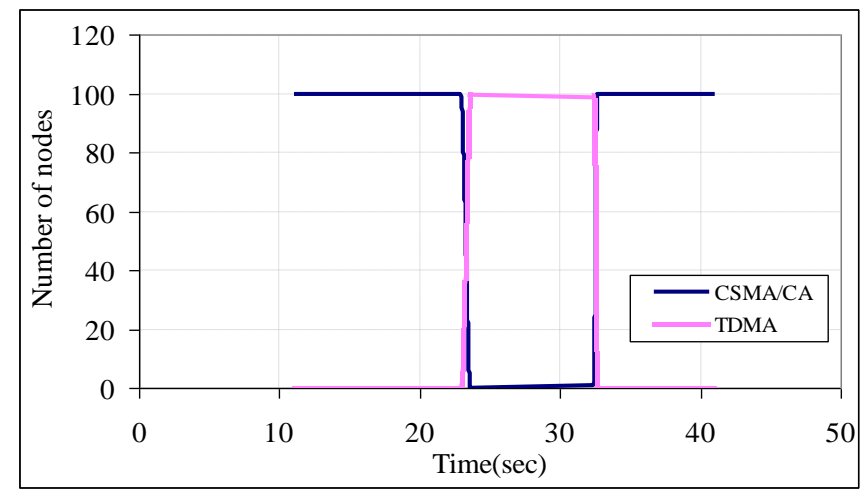

Fig. 9: MAC protocol dynamics with varying data rate 
The dynamic behavior of MAC switching is also shown in Fig. 9. As the data rate changes from high to low, the number of nodes running CSMA/CA reduces and the number of nodes running TDMA rises. Note that there is a lag between when the data rate changes and when the nodes actually switch their protocols. It was experimentally observed that this delay is caused due to the rate measurement latency at the MAC layer.

\subsection{Switching in Response to Number of Active Nodes}

As presented in Section 3.1, the number of active nodes in a neighborhood is the second key parameter that influences the quantity $\mu_{C S M A / C A}$, thus affecting the decisions for protocol switching. The variation of the number of active nodes in a network neighborhood is expected to trigger protocol switching for maintaining high network throughput.

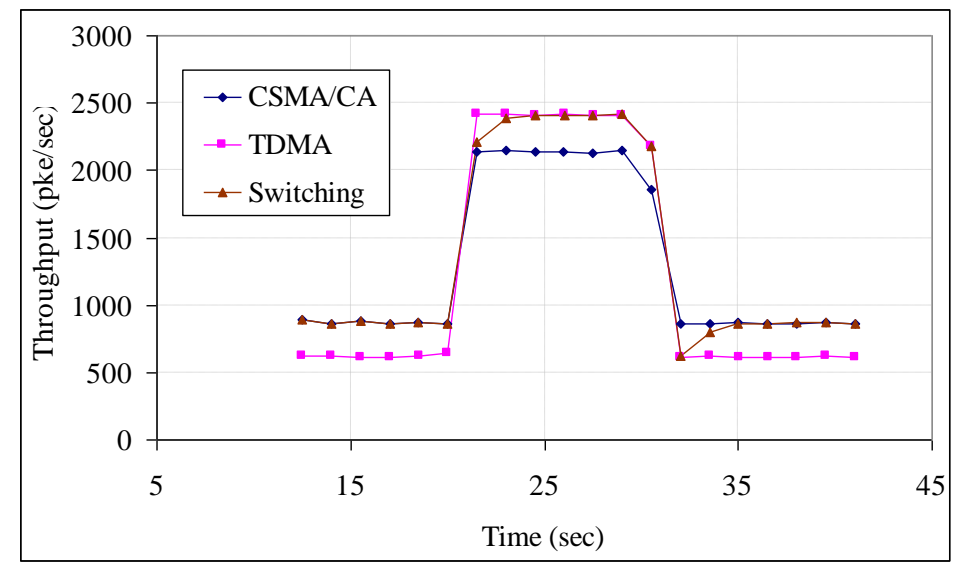

Fig. 10: Protocol switching with varying active node count

An experiment was conducted to study the impacts of variable number of active nodes on the performance of protocol switching. The throughput results in Fig. 10 correspond to an initial active node-count of 25 which is increased to 100 at time 21 second, and then changed back to 25 at time 31 second. When there are only 25 active nodes, they are randomly selected out of all 100 network nodes. All active nodes send data to all 1-hop neighbors at the rate of 35 packets/second.

Fig. 10 demonstrates that with lower number of active nodes (i.e. till time $21 \mathrm{sec}$ and then after 31 second) CSMA/CA performs better because of its larger MAC service rate in the absence of collisions as explained in Section 3.1. When the number of contender nodes increases, the contention brings down the service rate of CSMA/CA compared to that during the TDMA mode of operation. This explains why CSMA/CA outperforms TDMA in low active node-count situations, but not when it is high (i.e. between $21 \mathrm{sec}$ to $31 \mathrm{sec}$ ).

The throughput line representing protocol switching in Fig. 10 demonstrates that by exercising MAC layer protocol switching, the network is able to track the best possible throughput (those of CSMA/CA at low active node-count, and TDMA at high active node-count). This completes the validation of the switching logic presented in Section 3. 


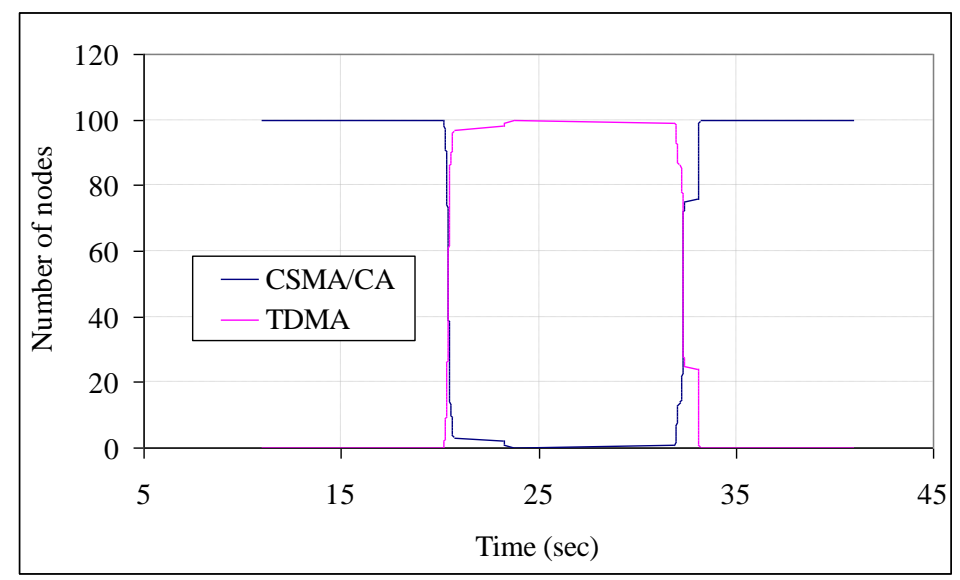

Fig. 11: Protocol dynamics with varying active node count

The dynamics of the number of nodes executing CSMA/CA and TDMA are depicted in Fig. 11. As in Fig. 9, the latency in switching transient is caused due to the rate measurement latency at the MAC layer.

\subsection{Switching with Spatially Clustered Traffic}

All experimental results so far describe protocol switching in a network with homogeneous traffic, thus causing all the active nodes to run the same protocol and to switch at the same time. In this Section we describe experiments with spatial traffic heterogeneity, which can cause different network nodes to run different MAC protocols at the same time, and to not necessarily switch in a synchronous manner.

Fig. 12 shows a rectangular sensor field equipped with a sensor network topology consisting of 400 nodes. Two different heterogeneous traffic profiles, each consisting of low, medium, and high density traffic regions, are used. In high density region, each node sends to all its 1-hop neighbors with a data rate of 3 packets/second per neighbor. Each node in the medium density region sends only to one of its 1-hop neighbors at a data rate of 140 packets/second. A node in the low traffic density region also sends only to one of its 1-hop neighbors at a rate of 12.5 packets/second. During a 60sec long experiment, the network traffic profile has been changed once from Profile-(1) to Profile-(2) at time approximately 31.0 second.

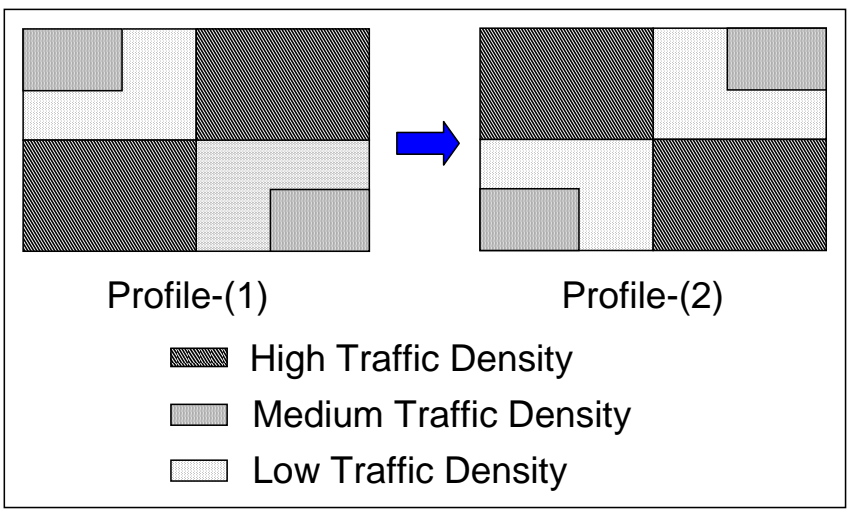

Fig. 12: Network with spatial traffic heterogeneity 
A node in the high traffic density region is expected to run TDMA to achieve higher throughput, because the MAC service rate of CSMA/CA is less than that of TDMA at high traffic level. CSMA/CA is expected in a medium traffic region, and TDMA is expected again in a low density region.

Fig. 13 reports the impacts of time-varying traffic profile on the throughput dynamics with and without MAC protocol switching. After the network is initiated at around $12 \mathrm{sec}$, observe that in the all-TDMA scenario, the network throughput settles down at around 18sec. Then it remains steady till $31 \mathrm{sec}$ which is when the traffic profile switches from Profile-(1) to Profile-(2). As a result of this, the throughput goes through a transient state before it settles back down roughly at the same value. Very similar trends can be also observed for the all-CSMA/CA scenario, except that with all-CSMA in this case the achievable network throughout happens to be higher than that of the all-TDMA case.

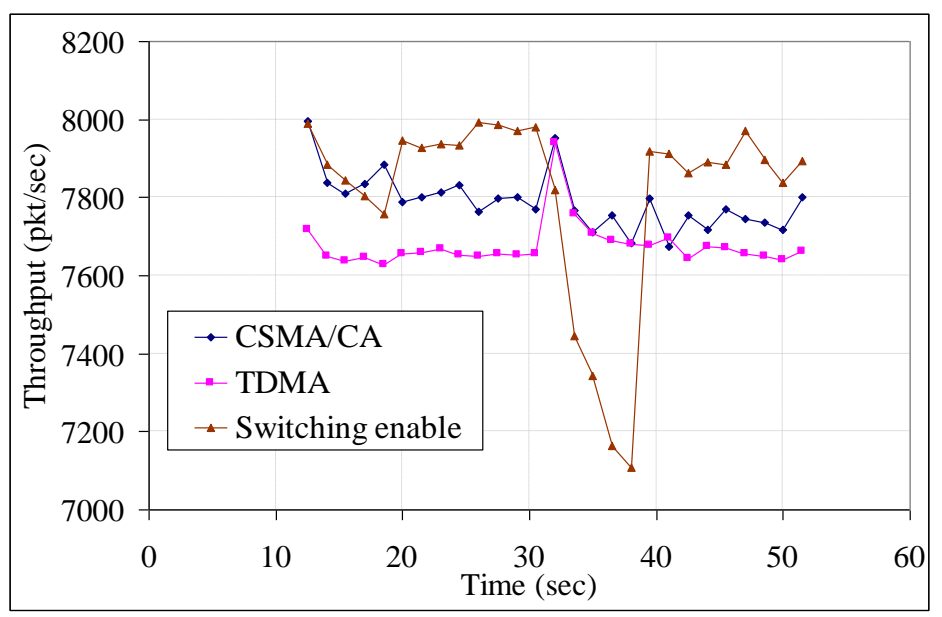

Fig. 13: Throughput dynamics with traffic heterogeneity

Observe that with protocol switching, the achieved throughput is higher than the two without switching cases during both before and after the traffic profile changes. Note that there is a significant transient drop for the with-switching case during the profile change. This drop in performance is caused because certain packets experience increased contention and collisions during the profile change. Such contentions and collisions lead to MAC layer drops and subsequent loss of throughput. After the transient situation is over, the protocol switching mechanism can track the new traffic profile distribution and able to select appropriate MAC protocols at the network nodes to bring the throughput back higher than the all-TDMA and all-CSMA/CA scenarios. 


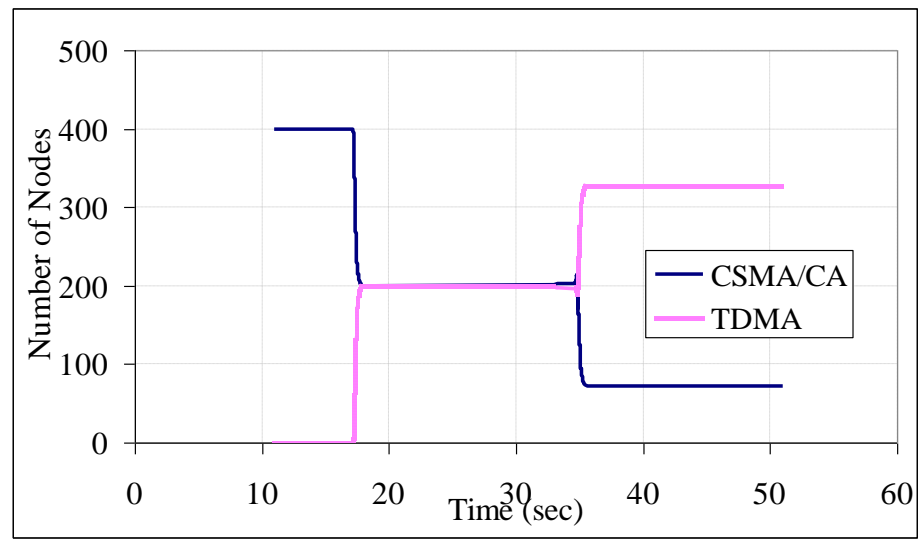

Fig. 14: Protocol dynamics with traffic heterogeneity

Two observations can be made from Fig. 14 which reports the number of nodes running TDMA and CSMA/CA during the entire experiment. First, nodes change their protocols in a period of few seconds after the traffic profile actually changes. This is due to the traffic rate measurement latency at the MAC layer. Second, the distribution of numbers of TDMA and CSMA/CA nodes during Profile-(1) and Profile-(2) are different. During when Profile-(1) is executed, the number of TDMA nodes and the number of CSMA/CA nodes are equal. However, during when Profile-(2) is executed, there are 328 nodes are running TDMA and only 72 nodes are running CSMA/CA. It was experimentally found that this variation is a result of the irregularity in network connectivity. Due to this topological irregularity, even nodes in the same traffic density region experience different number of contender nodes $n$, which impacts the protocol switching decision.

\subsection{Switching within a Fully Connected Network}

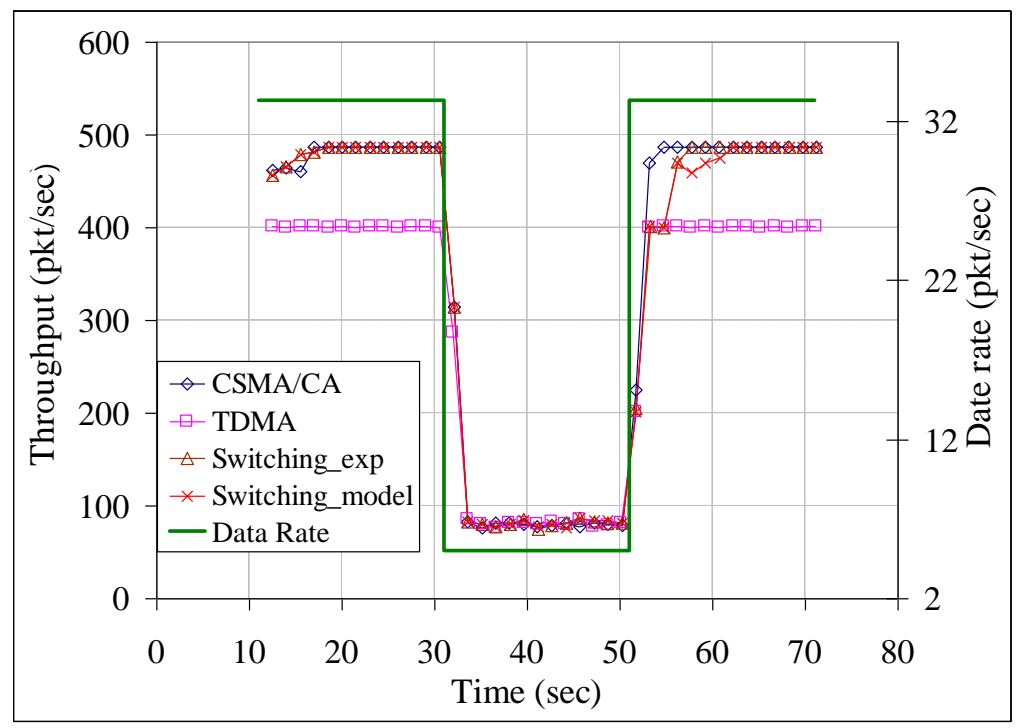

Fig. 15: Impacts of protocol switching decision thresholds in a fully connected topology

Fig. 15 shows the protocol switching results in a 20-node fully connected network with 16 active nodes sending 1-hop traffic with variable data rates. Traffic starts at time 11 second with a data rate of 33.3 packets/second, which changes to 5 packets/second at time 31 
seconds, and finally changes back to 33.3 packets/second at around 51 seconds.

As can be seen from the figure, while running TDMA at high data rates the MAC layer throughput is limited by its frame size. CSMA/CA, however, is able to achieve relatively higher throughputs at high rates. At lower rates, TDMA is expected to perform better than CSMA/CA because of its zero collisions. But the data rate of 5 packets/second is too low to demonstrate such benefits. Fig. 15 shows that like in the fully connected network scenario in Section 4.1, with protocol switching, the real-time throughput is generally able to track the upper envelop of both CSMA/CA and TDMA protocol at both high and low rate scenarios.

\section{Conclusions}

In this paper we have presented a distributed MAC protocol switching mechanism for maximizing MAC layer throughput in the presence of traffic and topology heterogeneity. The key idea behind dynamic MAC switching is for each node to use its local topology and traffic density information to decide the most suitable MAC protocol that can maximize the MAC layer throughout in the neighborhood. A formal MAC switching rule has been developed using analytical formulation of the MAC throughput available in the literature. NS2 based simulation experiments demonstrate that with the proposed MAC switching strategy, nodes in a sensor network are able to achieve maximum MAC throughput by adaptively choosing the appropriate MAC protocol in the presence of heterogeneity in terms of data rate and node population. Ongoing work on this topic includes generalizing the proposed mechanisms for a broader set of MAC protocols beyond CSMA/CA and TDMA.

\section{References}

[1] H. Balakrishnan, "Opportunities and challenges in high-rate wireless sensor networking," in 29th Annual IEEE International Conference on Local Computer Networks, 2004. , November 2004, http://dx.doi.org/10.1109/LCN.2004.91

[2] L. Kleinrock and F. Tobagi, "Carrier Sense Multiple Access for Packet Switched Radio Channels," in International Conference on Communications, Minneapolis, Minnesota, Jun. 1974, pp. 21B-1 -21B-7.

[3] W. Ye, J. Heidemann, and D. Estrin, "An Energy-Efficient MAC Protocol for Wireless Sensor Networks," in INFOCOM, June 2002, pp. 1567-1576, http://dx.doi.org/10.1109/INFCOM.2002.1019408.

[4] T. Dam and K. Langendoen, "An Adaptive Energy-Efficient MAC Protocol for Wireless Sensor Networks," in 1st ACM International Conference on Embedded Networked Sensor Systems, Nov. 2003, pp. 171-180, http://dx.doi.org/10.1145/958491.958512.

[5] Z. Chen and A. Khokhar, "Self organization and Energy Efficient TDMA MAC Protocol by Wake up for Wireless Sensor Networks," in 1st Annual IEEE Communications Society Conference on Sensor and Ad Hoc Communications and Networks 2004 (IEEE SECON' 04), Oct. 2004, pp. 335-341, http://dx.doi.org/10.1109/SAHCN.2004.1381934.

[6] V. Rajendran, K. Obraczka, and J. J. Garcia-Luna-Aceves, "Energy-Efficient, Collision-Free Medium Access Control for Wireless Sensor Networks," in International 
Conference on Embedded Networked Sensor Systems, Nov. 2003, pp. 181-192, http://dx.doi.org/10.1007/s11276-006-6151-z.

[7] I. Rhee, A. Warrier, J. Min, and L. Xu, "DRAND: Distributed Randomized TDMA Scheduling for Wireless Ad-hoc Networks," in the 7th ACM international symposium on Mobile ad hoc networking and computing, Florence, Italy, May 22-25, 2006, http://dx.doi.org/10.1145/1132905.1132927.

[8] G.-S. Ahn, E. Miluzzo, A. T. Campbell, S. G. Hong, and F. Cuomo, "Funneling-MAC: A Localized, Sink-Oriented MAC For Boosting Fidelity in Sensor Networks," in Fourth ACM Conference on Embedded Networked Sensor Systems (SenSys 2006) Boulder, Colorado, USA, November 2006.

[9] Funneling-MAC

Technical

Report:

http://www.cs.dartmouth.edu/ sensorlab/funneling-mac/TAPTR-2006-08-003.pdf."

[10] J. V. Sudarev, L. B. White, and S. Perreau, "Performance Analysis of 802.11 CSMA/CA for Infrastructure Networks under Finite Load Conditions," in The 14th IEEE Workshop on Local and Metropolitan Area Networks, 2005. LANMAN 2005. September 2005, http://dx.doi.org/10.1109/LANMAN.2005.1541535.

[11] M. Y. Chung, M.-H. Jung, T.-J. Lee, and Y. Lee, "Performance Analysis of HomePlug 1.0 MAC With CSMA/CA," IEEE Journal on Selected Areas in Communications, vol. 24, pp. 1411-1420, July 2006, http://dx.doi.org/10.1109/JSAC.2006.874404.

[12] W. Lee, C. Wang, and K. Sohraby, "On Use of Traditional M/G/1 Model for IEEE 802.11 DCF in Unsaturated Traffic Conditions," in IEEE Wireless Communications and Networking Conference (WCNC' 06), Las Vegas, Nevada, April 2006, pp. 1933-1937, http://dx.doi.org/10.1109/WCNC.2006.1696591.

[13] Y. Barowski and S. Biaz, "The Performance Analysis of IEEE 802.11 Under Unsaturated Traffic Conditions, ftp://ftp.eng.auburn.edu/pub/techreports/csse/04/CSSE04-08.pdf," August 2004.

[14] G. Bianchi, "Performance Analysis of the IEEE 802.11 Distributed Coordination Function," IEEE Journal on Selected Areas in Communications, vol. 18, pp. 535-547, March 2000, http://dx.doi.org/10.1109/49.840210.

[15] O. Tickoo and B. Sikdar, "A queueing model for finite load IEEE 802.11 random access MAC," in IEEE International Conference of Communication (ICC), 2004, pp. 175-179, http://dx.doi.org/10.1109/ICC.2004.1312475.

[16] "The network simulator: NS-2, at http://www.isi.edu/nsnam/ns.

[17] F. Yu, T. Wu, and S. Biswas, "Towards In-Band Self-Organization in Energy-Efficient MAC Protocols for Sensor Networks", IEEE Transactions on Mobile Computing, vol. 7, no.

2, pp. 156-170, http://dx.doi.org/10.1109/TMC.2007.70719.

\section{Copyright Disclaimer}

Copyright reserved by the author(s).

This article is an open-access article distributed under the terms and conditions of the Creative Commons Attribution license (http://creativecommons.org/licenses/by/3.0/). 